\title{
Directional cues from CXCR4
}

The CXC-chemokine receptor 4 (CXCR4) provides essential signals that guide thymocytes on their developmental journey through the thymus, according to a recent study in The Journal of Immunology.

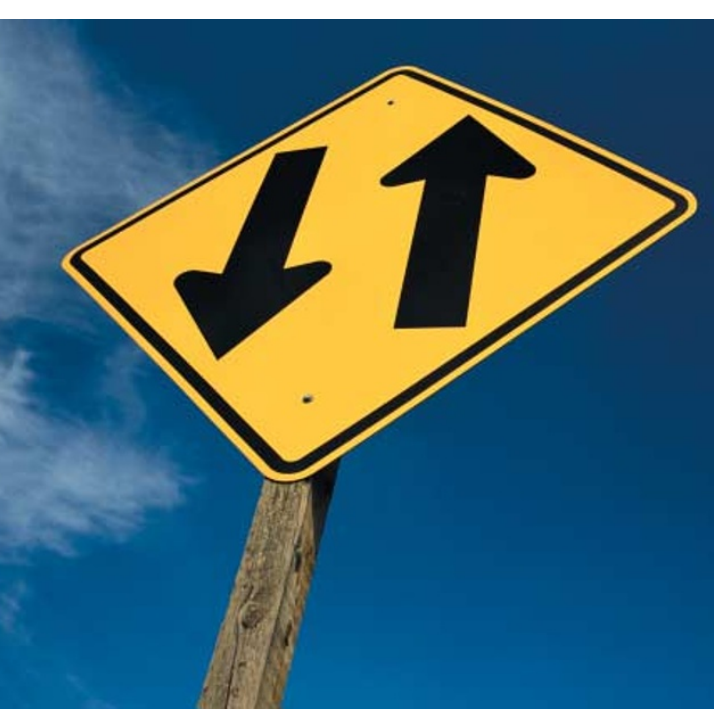

As thymocytes mature in the post-natal thymus, they migrate from their entry point near the cortico-medullary junction (CMJ), outwards across the cortex towards the capsule, and then back again across the cortex towards the medulla. This migration enables these developing progenitor cells to interact with thymic stromal cells that provide signals that are required for commitment to the T-cell lineage, and for efficient thymocyte differentiation and proliferation.

How is this cortical migration controlled? Petrie and colleagues investigated the role of chemokines in this process by first asking which chemokine receptors were expressed by thymocyte progenitors. Of all of the known chemokine receptors, CXCR4 was the most abundant, being expressed by all thymocyte prognenitors. Further experiments showed that the ligand for this receptor, CXCL12, is produced by cortical stromal cells, indicating that signals through CXCR4-CXCL12 could potentially be involved in guiding progenitors into the cortex.

To investigate this possibility, the authors next measured whether thymocyte progenitors

mouse CTLA4 induced IFN- $\gamma$ production, upregulation of IDO expression and tryptophan degradation by mouse DCs. They then showed that regulatory $\mathrm{T}$ cells also induced IFN- $\gamma$ production and tryptophan degradation by DCs and that this was inhibited by CTLA4-specific neutralizing antibodies. Activation of regulatory $\mathrm{T}$ cells with CD3-specific antibodies enhanced their ability to induce IFN- $\gamma$ production and tryptophan degradation by DCs and this depended on their increased expression of CTLA4.

Does tryptophan catabolism alter the capacity of DCs to induce immune responses in vivo? The ability of CD8- DCs loaded with a synthetic peptide (NRP-A7) to induce a persistent immune response in mice was eliminated by pre-exposure of the DCs to regulatory T cells activated with CD3-specific antibody. However, the ability of peptide-loaded DCs to initiate an immune response was restored if an inhibitor of IDO was present during DC exposure to the activated regulatory T cells.

These studies identify a CTLA4dependent mechanism by which $\mathrm{CD} 4{ }^{+} \mathrm{CD} 25^{+}$regulatory $\mathrm{T}$ cells induce immunosuppression - modulation of could migrate in response to CXCL12. Transwell migration assays using CXCL12 as a chemoattractant showed that all populations of progenitors that were tested migrated towards CXCL12. To confirm the role of CXCR4 signalling in this directional movement, the in vivo migration of CXCR4-deficient progenitor thymocytes was assessed. T-cell numbers were low and thymocyte differentiation was blocked at an early stage in mice reconstituted with bone marrow that lacked CXCR4 expression. Furthermore, thymocytes derived from CXCR4deficient bone marrow accumulated at the CMJ and did not migrate efficiently into the cortex.

This study highlights the essential and nonredundant role for CXCR4-CXCL12 signalling in controlling the migration of thymocyte progenitors across the cortex - a process that is required for the development of mature T cells in the post-natal thymus.

Jenny Buckland

\section{(2) References and links}

ORIGINAL RESEARCH PAPER Plotkin, J. et al. Critical role

for CXCR4 signaling in progenitor localization and T cell differentiation in the postnatal thymus. J. Immunol. 171, 4521-4527 (2003)

FURTHER READING Petrie, H. T. Cell migration and the control of post-natal T-cell lymphopoiesis in the thymus. Nature Rev. Immunol. 3, 859-866 (2003)

\section{WEB SITE}

Howard Petrie's lab: http://www.mskcc.org/

mskcc/html/11136.cfm

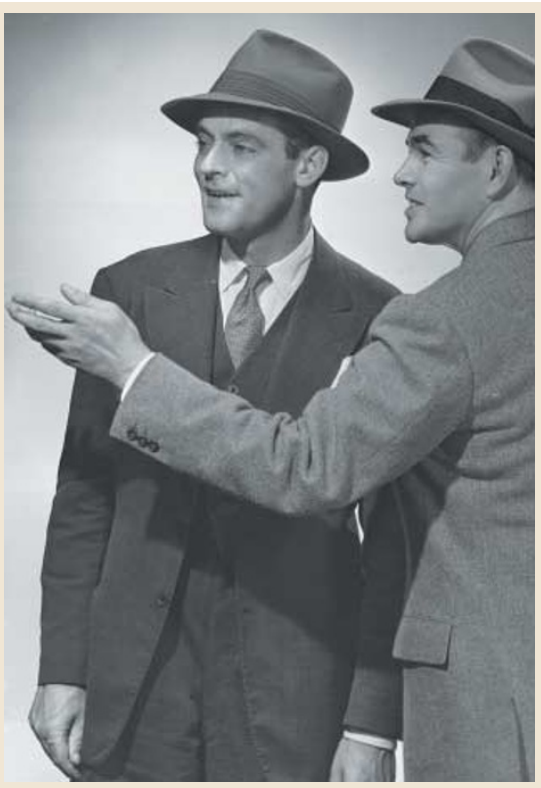

DCs to initiate IFN- $\gamma$ production and, thereby, IDO activity and tryptophan catabolism.

Karen Honey

(2) References and links ORIGINAL RESEARCH PAPER Fallarino, F. et al. Modulation of tryptophan catabolism by regulatory $T$ cells Nature Immunol. 26 October 2003 (doi:10.1038/ni1003) 